at the photocathode, and $m=\mathbf{I}$. It follows that the maximum allowable exposure is about rooo sec.

The maximum exposure time set by thermal electrons can be increased by a factor of Ioo by reducing the temperature to that of solid $\mathrm{CO}_{2}$, namely, $-80^{\circ} \mathrm{C}$. The refrigeration of photocathodes is already common practice in photo-electric photometry. Since the thermal emission of cesium-oxide-on-silver photocathodes (sensitive to the infra-red) is much greater than that of cesium-antimony cathodes, refrigeration is certain to be essential wherever infra-red cathodes are employed.

It is obvious from the preceding two papers that image tubes will be considerably larger and heavier than typical plate-holders for a spectrograph. The image converter with plate-exchanger as under development at Yerkes Observatory has a weight of about $5 \mathrm{~kg}$. plus refrigeration equipment that may add another $2 \mathrm{~kg}$. Overall dimensions are of the order of 20 to $30 \mathrm{~cm}$. While some spectrographs would need to be considerably redesigned to accommodate heavy and bulky loads of that type in place of conventional plate-holders, others such as the Cassegrain spectrograph at the McDonald Observatory and the coudé spectrographs elsewhere can adapt an image converter with only minor alterations.

The adaptation of image converters to small fast spectrograph cameras should not be difficult if one additional reflection is introduced. Then the cathode can be placed either at the centre of the mirror or at the side of the optical bundle. With a design of this type, the whole camera in some cases may have to be refrigerated. As pointed out above, the image converter provides an independent means of altering the speed of a camera by electron-optical demagnification.

If the anticipated gain of image tubes can be realized in practice, the new fields of research open to the stellar spectroscopist seem limited only by the imagination of the observer. One obvious field of application is the observation of red-shifts of faint extragalactic nebulae where the discrimination of the nebular spectrum from that of the night air-glow must be improved. Other obvious applications include higher dispersion spectra of moderately faint objects (particularly in the infra-red region), low-dispersion spectra of stars which are too faint to be reached by present conventional methods at all, and the spectroscopic observation of rapidly varying phenomena. The two-dimensional spectral classification of stars down to I6th or I7th magnitude should provide opportunities in the study of galactic structure not dreamed of a decade ago. The classification and analysis of spectra might even be extended to the brighter stars of neighbouring galaxies.

In some applications, such as high-dispersion spectra, the full gain of roo in speed can be anticipated, but in some other applications, such as the determination of line intensities or the discrimination of nebular spectra, the gain manifests itself as a ten-fold (square root of I0o) increase in accuracy or detectability. In any case, it appears to be a safe prediction that image tubes will stimulate new life in stellar spectroscopy.

\title{
5. THE APPLICATION OF IMAGE TUBES TO THE PHOTOGRAPHY OF PLANETS
}

\author{
By Russell Morgan, Ralph Sturm and Albert Wilson
}

\section{The Lowell Observatory and the Laboratory of Astrophysics and Physical Meteorology of the Johns Hopkins University}

The advantages of photographic observations over visual observations are unequivocal in such fields as astronomical photometry and spectroscopy, but up to the present time it has been impossible to record photographically all of the fine detail of planetary features which the human eye has been able to detect. In the case of the planet Mars, for example, the failure of photographs to record the detail which can be observed 
visually has led to widespread controversy over the reality of some of the fine surface markings. The primary reason for the discrepancy is that the human eye and a photographic plate respond differently to an image which is turbulently indistinct. The human eye stores light for a small fraction of a second and relays a series of impressions or pictures to the brain, which in turn selects and remembers a sub-series of pictures (such as those which have good definition) and discards the remainder. The photographic plate, on the other hand, possesses no mechanism of selectivity but integrates over blurred and clear intervals together. Since the eye and the photographic plate perform unlike operations, it is not surprising that their records are different. With the development of image-tube techniques, however, there is now the possibility of obtaining enhanced photographs by simulating the visual process of selecting a sub-set of good images from a series of very short exposures.

The property of an image intensifier which makes such an operation possible is the increased efficiency of its photo-sensitive surface over that of the photographic plate. During the exposure times usually required for conventional photography of a planet, the dancing image has an opportunity to make several excursions, whereas with an image intensifier the exposure times can be made short enough for the effects of image turbulence to be greatly reduced. To determine how much advantage may be expected from shorter exposure times, it is first necessary to understand the nature of the disturbances affecting the telescopic image which is to be recorded.

This difference in definition between what may be observed visually and photographically by a telescopic system is not evident when objects are observed close at hand. For example, with the same optical systems, there is just as much detail on a photograph of the image of a 'resolution pattern' when not photon-limited as is detectable visually in the aerial image of the pattern. This means, of course, that the disturbance causing the difference in quality between visual and photographic images in the case of distant objects is a factor external to the optical-photographic system, namely, atmospheric turbulence or 'seeing'.

There are several effects produced in a telescopic image by atmospheric seeing. Principally these are: the translatory motion, change in sharpness, change in brightness, and change in form. These restless modifications of the image can occur very rapidly and often are so extreme that the true nature of the image is unrecognizable. At other times the effects of seeing are very slight and occur slowly. It is only at these latter moments of good seeing that good planetary photographs can be made. But even under the optimum conditions of seeing the image is never completely at rest or completely free of changes. Experiments conducted over many years with emulsions, filters, image scale, telescope aperture, etc., have resulted in values giving optimum definition, and the conclusion is that the quality of planetary photographs is not limited by these factors but primarily by atmospheric seeing.

In order to understand quantitatively the problems of seeing as they apply to planetary photography and to determine whether steps might be taken to overcome some of the deleterious effects of seeing, studies were undertaken at the Lowell Observatory with a specially designed seeing recorder. The recorder consisted of a Du Mont type 321 oscillograph camera using $35 \mathrm{~mm}$. movie film which could be driven at constant speeds from $2 \mathrm{~cm}$. per min. to go metres per min. This camera was mounted at the focus of a telescope and a chopper was interposed in the light path to break the trailed star image into discrete segments for ease of measuring. Very briefly the results were as follows:

(I) The amplitude and rate of translatory motion were studied. It was found that even under conditions of good seeing, the image moved as much as a second of arc in an interval of less than one-tenth of a second of time. It is quite evident that in the presence of motions of this sort it would be impossible to photograph in a conventional manner any of the fine surface detail on the planet Mars for which exposures of one second or more are typical.

(2) The sharpness of the 'instantaneous' image was studied. It was found that images of $\mathrm{I} / \mathrm{I} 2 \mathrm{O} \mathrm{sec}$. duration varied in sharpness to a degree comparable with their variation in 
position. During very good seeing when excursions were limited to, say, $0.5 \mathrm{sec}$. of arc, the image formed by a telescope of 9 in. aperture was found to be near the optical limit of sharpness only about one-fourth of the time. It was further found that the definition was a function of the aperture; the larger the aperture, the less frequent was the occurrence of good images.

(3) The distortion of the image was studied. This was done by observing the separation of the components of a double star ( $\alpha$ Capricorni, 6 min. of arc apart) and correlating the constancy of the separation with the quality of the seeing. Under conditions of good seeing, this separation was more nearly constant than the stationarity of the images. This would mean that any line-segment joining two points on a planetary image should maintain fairly constant length and that the disk should not experience severe distortions.

In short, two basic conditions must be met in order to secure good photographs of a planet: $(a)$ the telescope aperture must be small enough so that the image is not multiple, broken, or 'soft'; that is, the effect of seeing must be limited mainly to excursions of the whole image as a unit. (b) The exposure time must be limited to a very small fraction of a second (a few hundredths of a second at most) in order that there is a reasonable probability of catching an occasional exposure during which the motion of the image is very much less than its full excursion amplitude. These two conditions oppose one another; the more that either is favoured, the less well can the other be met. The present situation is that ordinary unaided photography is unable to meet these conditions favourably for even the brightest planets, but the advantage in quantum efficiency of photocathodes over photographic plates should provide the additional sensitivity needed to meet both conditions quite well.

An opportunity to test the foregoing conclusions was presented in the summer of 1954 by the development of an image-orthicon intensifier for use on faint objects by the Radiological Laboratory of Johns Hopkins Hospital. The original purpose of the equipment was the intensification of fluoroscopic images to facilitate diagnostic X-ray observation of movements within the human body. In its original application, this intensifier uses an $f / 0.75$ lens of IIo $\mathrm{mm}$. focal length to relay the image from a fluorescent screen to the photo-electric surface of an image-orthicon tube. The electrical output of this tube is amplified by a specially designed low-noise amplifier, and an intensified image is displayed on a $25 \mathrm{~cm}$. kinescope screen where it, rather than the image on the original fluoroscope screen, is photographed. The display is comprised of roz 9 interlaced scanning lines from a $25 \mathrm{~mm}$. orthicon raster having a frame repetition rate of 30 per sec.

Under a contract with the Office of Naval Research, the Laboratory of Astrophysics and Physical Meteorology of Johns Hopkins University arranged for this image intensifier to be tested for planet observations at the Lowell Observatory. During the opposition of Mars in July of 1954 the image intensifier was mounted on the Lowell 24 in. refractor to photograph the Martian surface and atmosphere.

Various lens systems were used to focus the telescope image upon the photocathode of the orthicon. The best definition was obtained with a positive relay lens giving a I0.5 mm. image of Mars (whose apparent diameter was about $20 \mathrm{sec}$. of arc) on the photocathode which provided for 425 scanning lines acroșs the disk. The apparatus had an electronic scale-amplification factor of $7 \cdot 2$, giving a $75 \mathrm{~mm}$. image of Mars on the kinescope. The kinescope screen was photographed with an $f / \mathrm{x} \cdot 5$ Leica giving a final image diameter of $14 \mathrm{~mm}$. Because of the interlaced sweep and focal plane shutter, I/I5 sec. was the minimum usable exposure. Considerably shorter exposures would have been possible if it had not been necessary to insert a Wratten $25 \mathrm{~A}$ red filter in front of the photocathode in order to obtain the best discrimination of surface detail on Mars; the image orthicon has a cesium-antimony photosurface whose maximum spectral sensitivity is around $4300 \AA$. These photographs clearly show the Solus Lacus region with the Nectar Canal discernible on many of the negatives. Although obtained during mediocre seeing, they are only a little short of competing with the best direct photographs.

In addition to increased sensitivity, a second advantage of the image intensifier for planetary observations is the control over contrast. The intensifier has two contrast 
controls, one which adjusts the gain and the other which introduces a bias to adjust the zero value of intensity (see paper no. 2). In spanning the gap between what may be observed visually and what may be recorded photographically on planets, these contrast adjustments have proved to be of considerable value.

The first experiments suggested techniques which should improve future tests. One procedure would be to place a shutter having speeds up to I/200 sec. in front of the photocathode and to synchronize it with a 'suppressor circuit' which keeps the kinescope screen dark except during the frame scan immediately following the exposure of the cathode. The camera used to photograph the kinescope screen can then operate continuously with an open shutter.

With an image-tube system having a larger number of frame scans, a more suitable spectral response, and a synchronized shutter, it should be possible to overcome present limitations and to surpass any planet photographs thus far obtained.

\section{SOME APPLICATIONS OF IMAGE CONVERTERS IN THE U.S.S.R.}

By V. I. Krassovsky, Geophysical Institute of the Academy of Sciences, U.S.S.R.

In order to photograph astronomical objects of extremely small intensity or to obtain their spectra, astronomers are obliged to use not only large telescopes and photographic materials of the highest sensitivity, but also extremely long exposures sometimes lasting many hours. If the application of image converters is aimed at detecting undiscovered objects of extremely low intensity, it is also necessary that converters should be capable of long exposures. An increase in sensitivity, without provision for prolonging exposures, may only shorten the exposure times for already known faint objects or their spectra. For astronomical investigations it is also highly desirable to extend the spectral sensitivity, particularly into the infra-red.

Various photo-electric methods were introduced into astronomical practice during the past decade. Their principles, their main characteristics, and the properties of photocathodes are sufficiently described in the literature. It might, therefore, be advisable to recall here certain of their peculiarities which determine their role in astronomical investigations.

As is known, the slow fall of sensitivity in the long wave-lengths in the case of cesiumoxide-on-silver photocathodes is extremely valuable. It does not exceed an order of magnitude between the visible region and about I $\mu$, whereas the sensitivity of the best photographic emulsions decreases several orders of magnitude in that range.

The possibility of a considerable reduction in size by means of electron-optical demagnification inside an image converter permits shortening exposures considerably. This is equivalent to an increase in the focal ratio, which cannot be realized by means of ordinary optical methods(r). This method is advisable in a number of cases, in spite of the resulting diminution in the distinctness of images.

Artificial cooling of the photocathode (for example, by means of solid carbon dioxide) decreases the dark current to such an extent that exposures of several tens of hours become possible(r). The generation and use of high accelerating voltages of $15-20 \mathrm{kV}$. is quite feasible. The production of fine-structure fluorescent screens of good quality, the emission of which is matched to the spectral response of photographic emulsions, meets no insurmountable difficulties.

However, all photo-electric image-converter systems possess a number of essential deficiencies. The most important restriction on the application of image converters is the small size of the working field when flat cathodes are used. The reception of stationary images is hampered when photo-electric image converters of long dimensions are used; the shift of images is caused in the first place by the external magnetic field, which is particularly evident when a parallactic arrangement of the instrument is used. Ordinary photo-electric image converters permit resolving on the photocathode about $20-40$ line 